Cahiers de recherches médiévales

Journal of medieval studies

2 | 1996

Regards sur le Moyen Âge

\title{
Mélusine et Geoffroi à la grand dent
}

Apories diégétiques et réécriture romanesque

\section{Claudio Galderisi}

\section{CpenEdition}

Journals

Édition électronique

URL : https://journals.openedition.org/crm/2484

DOI : $10.4000 / \mathrm{crm} .2484$

ISSN : 1955-2424

Éditeur

Honoré Champion

Édition imprimée

Date de publication : 12 décembre 1996

Pagination : 73-84

ISSN : 1272-9752

Référence électronique

Claudio Galderisi, « Mélusine et Geoffroi à la grand dent », Cahiers de recherches médiévales [En ligne],

2 | 1996, mis en ligne le 04 février 2008, consulté le 15 décembre 2022. URL : http://

journals.openedition.org/crm/2484; DOI : https://doi.org/10.4000/crm.2484 


\section{RM}

\section{Mélusine et Geoffroi à la grand dent. Apories diégétiques et réécriture romanesque.}

Tout ce qui est dit est cuit.

Langage toujours trop tard venu sur nous même.

A la fin du XIV siècle la littérature en prose semble de plus en plus en équilibre instable entre l'évasion complaisante et autoréférentielle dans l'histoire, avec une représentation des valeurs fondées sur le passé, et le récit court qui, de par sa structuration, se situe tout entier ou presque dans le présent, mettant en cause les relais culturels autour desquels s'articule la dynamique sociale de ce même présent, mais dans un cadre qui reste celui de la fiction, et qui est, comme nous le verrons à travers l'analyse des déliaisons structurelles opérantes dans le Roman de Mélusine, soumis à des sollicitations formelles aussi bien infra qu'extradiégétiques.

Toute cette production témoigne, en effet, de la propension du Moyen $\mathrm{Age}^{2}$ à son automne à regarder intensément dans son passé, pour chercher à s'annuler dans son propre reflet, dans sa réfraction littéraire. Ce sont, donc, les valeurs du passé, valeurs mythiques, ou plutôt pensées mythiques, souvent, qui donnent au temps une épaisseur, une profondeur qui grisent l'homme médiéval, lui procurent un sens de vertige et en même temps de nausée qui aboutit à une forme de dédoublement ontologique, dont la littérature nous offre dans un miroir convexe le digramme surchargé: un univers noble et pur, mais aussi sclérosé et improbable dans son anachronique et précieuse intégrité idéologique, sous lequel point un autre agressif, intolérant, vulgaire, chaotique et rusé, intellectuellement irrecevable, et cependant littérairement stimulant et vital.

Comme on l'a souvent dit, cet homme découvre en même temps le vieillissement des modes et de la langue, vieillissement qui est mis en évidence par la diffusion de la lecture individuelle, et plus généralement par l'utilisation d'un français moyen qui sert d'élément de comparaison, outre que de communication. Mais il découvre aussi que, toute surannée et archäque qu'elle est, l'idéologie courtoise est la seule qu'il ait bâtie. De ce double constat, apparemment contradictoire, nait la nouvelle mission de la prose : en la nommant créer une réalité qui serve de miroir à toute une société.

Ainsi, l'autre réalité créée par l'écriture romanesque, l'ailleurs narratif, représente souvent les contours d'un monde insouciant de son propre déclin, qui se met en scène dans ses plus intimes, et souvent fantastiques, détails, avec l'espoir secret qu'une telle représentation offre aux futures générations - c'est-à-dire aux futurs lecteurs - l'image d'un

\footnotetext{
${ }^{1}$ Pascal Quignand, La leçon de musique, Paris, Hachette, 1987, p. 27.

${ }^{2}$ Ou plutôt des moyens âges, car il semble bien qu'à « la tentation d'une préhistoire » (cf. Michel Zink, Le Moyen Age et ses chanson, Paris, Editions de Fallois, 1996, pp. 135-38), à laquelle ont cédé les modemes, n'ont pas non plus échappé les différentes périodes de ce qu'il est convenu d'appeler le Moyen Age.
} 
Age qui n'est plus, parce qu'il n'a jamais été, et qui pourtant enfin est. Et cela parce qu'il hante l'imaginaire collectif finissant par boucler le " cercle herméneutique de la narrativité et de la temporalité "' "se posant comme une mimèsis à l'envers, jusqu'à interférer avec les coondonnées spatio-temporelles du monde réel ${ }^{4}$. Ainsi, les différents modes de représentation des rites du passé deviennent le modèle d'une société toute penchée sur son fantasme, dans une crispation du temps qui laisse enfin au temps de l'écriture tout l'espace du jeu, mais un espace désormais subtilement perçu comme déformé, étriqué, «vicieux », aussi inadéquat, sinon plus, que celui quotidien d'où cet homme cherche à s'évader. La prose devient alors pour la première fois non seulement l'étalon qui mesure le degré de cohésion culturelle de la société, mais aussi un puissant instrument de redéfinition du réel, de tout le réel, historique et narratif. Le réalisme double de vérité et d'historicité, dont la prose était issue, dévoile le masque de son hypocrisie, et montre le visage de la subjectivité: subjectivité de l'homme, de l'artiste, mais aussi subjectivité d'une société, qui aspire à la survie par la mise en représentation de ses valeurs et de ses fonctions littéraires. Il n'est pas étonnant, alors, de voir fleurir toute une série de mises en prose d'anciens romans en vers. Ce principe de la réécriture, qui semble supposer une idée de modemité littéraire, implique en réalité une immuabilité du référent littéraire, c'est-à-dire de l'objet du récit, cristallisé à travers le filtre de l'histoire. Mais la pression du milieu, et celle du mécène en premier, favorisent le roman généalogique écrit sur commande à la gloire d'une famille ou d'un personnage, comme c'est le cas pour Mélusine. Au début du $\mathrm{XV}^{e}$ siècle l'articulation esthétique entre histoire et roman est désormais chose faite, donc, et comme l'histoire racontée a pour objet aussi d'enseigner des leçons, non seulement la fiction se voit affublée de l'habillage de la chronique, mais elle est en plus soumise à une intention didactique et pédagogique, qui était aussi, d'ailleurs, un des traits génétiques de la prose à ses débuts.

Mais ces formes que prend la parole, ces récits pleins de gestes du passé regardent inexorablement vers le présent, et se révèlent comme autant de failles culturelles où peut enfin faire surface et s'exprimer toute l'énergie de survie qui s'est dégagée de cette société. Après s'être docilement pliée, par un mouvement de retour mimétique, la parole heurte, grince, dissémine, comme dans « une virtuelle trainée de feux sur des pierreries ${ }^{5}$ », les signifiés enfin mobilisés par l'anachronisme du contexte (tout le contexte ${ }^{6}$ ). Elle ouvre de nouvelles pers-

\footnotetext{
${ }^{3}$ Paul Ricour, Temps et récit. 1. L'intrigue et le récit historique, Paris, Seuil, 1983, p. 138.

"Comme le rappelle Daniel Poirion, «ce sont déjà des œuvres littéraires, et non des modèles abstraits, des archétypes, qui servent de matrice à l'engendrement de l'espace textuel » (Daniel Poirion, «Ecriture et réécriture », Ecriture poétique et composition romanesque, Orléans, Paradigme, 1994, p. 461).

${ }^{5}$ Stéphane Mallarmé, Euvres complètes, éd. d'Henri Mondor et G. Jean-Aubry, Paris, Gallimard (La Pléiade), 1951, p. 366. J'ai utilisé ailleurs cette image mallarméenne, déjà reprise par Julia Kristeva dans La réwolution du langage poétique (Paris, Seuil, 1974, p. 272), pour évoquer le phénomène de "transsubstantiation de la structure syntaxique innée" (Claudio Galderisi, Le lexique de Charles d'Ortéans dans les Rondeaux, Genève, Droz, 1993, p. 89), mais je crois qu'on peut y percevoir aussi l'intuition d'une entropie négative de la chose écrite, telle qu'elle semble se dessiner comme conséquence de la rupture des codes d'énonciation, ou de réception.

"On peut très bien adopter le "théorème " énoncé par Daniel Poirion à propos du roman Jehan de Saintré, et dire avec lui qu'il n'y a pas de véritable mimèsis dans un roman historique, « mais une falsification du contexte pour fabriquer un monde référentiel en trompe-l'œil." (Daniel Poirion, op. cit. p. 459).
} 
pectives auto et endoscopiques, qu'éclaire et prolonge la différence ontologique. C'est ce terrain instable d'une forme nouvelle et déjà décadente dans ses présupposés esthétiques que privilégie l'ironie pour s'infiltrer et corrompre enfin la perception du discours, l'aperception du monde, altérant par là même, non seulement l'intentio auctoris et l'intentio lectoris, mais jusqu'à l'intentio operis' .

Or, c'est la structure diégétique du récit, ou plutôt son muthos, c'est-à-dire l'agencement des faits en système cohérent $t^{8}$, qui doit prendre en charge cette « déperdition du sens ${ }^{9}$ " raccorder ces étirements impossibles de la logique narrative dans son appréhension du monde et du moi. Cette cicatrice culturelle cousue du fil blanc du romanesque révèle plus qu'elle ne cache, alors, la fracture bio-anthropologique temps-espace ${ }^{10}, l^{\prime}$ impossible coexistence diégétique entre conscience du monde, du présent, et pensée mythique du soi, entre la « révolte contre le temps concret, historique [et la] nostalgie d'un retour périodique au temps mythique des origines, au Grand Temps ${ }^{11}$. » Mais sur le plan de la représentation narratologique, elle prélude à la naissance d'une nouvelle esthétique de la forme brève, qui en France ne trouvera sa codification que vers la moitié du $X v^{e}$ siècle.

Parmi ces mythes autour desquels se construit pour l'homme du Moyen Age le puzzle de son identité culturelle, celui de Mélusine semble pouvoir illustrer exemplairement cette nécessité de conjuguer histoire et mythe, merveilleux et chevalerie, chrétienté et dimension surnaturelle, fantastique et politique pour construire une identité que la mise en intrigue finit par faire voler en éclats.

Déjà au XVW" siècle, en plein essor de la Bibliothèque bleue de Troyes, l'histoire de Mélusine était perçue comme «l'histoire non pas d'un personnage mais d'une idée, d'un

\footnotetext{
'J'utilise ici ces termes surtout dans l'acception proposée par Umberto Eco (cf. Les limites de l'interprétation, Paris, Le Livre de Poche, 1992, pp. 29-32).

${ }^{8} \mathrm{~L}$ 'acception que je propose ici de cette catégorie aristotélicienne est celle déjà avancée par Paul Ricoeur (cf.op. cit. p. 69).

${ }^{9}$ Michel Zink, Litternature francaise du Moyen Age, Paris, PUF, 1992, p. 336.

${ }^{10}$ On pourrait ici utiliser les concepts augustiniens de intentio et distentio. La tension qui lie intentio et distentio est une tension spéculative inhérente à tout acte poétique de mise en intrigue. Relisons l'exemple-paradoxe proposé par Augustin : « Je me prépare à chanter un chant que je connais. Avant que je commence, mon attente se tend vers l'ensemble de ce chant ; mais quand j'ai commencé, à mesure que les éléments prélevés de mon attente deviennent du passé, ma mémoire se tend vers eux à son tour ; at les forces vives de mon activité sont distendues, vers la mémoire à cause de ce que j'ai dit, et vers l'altente à cause de ce que je vais dire. Néanmoins mon attention est là, présente ; et c'est par elle que transite $œ$ qui était futur pour devenir passé. Plus cette action avance, avance, plus s'abrège l'attente et s'allonge la mémoire, jusqu'à ce que l'attente toute entière soit épuisée, quand l'action toute entière est finie et a passé dans la mémoire. " (Euvres de saint Augustin, trad. de E. Tréhorel et G. Bouissou, Paris, Desclée de Brouwer, t. XIV, 1962, livre XI, paragraphe XXVIII). C'est dans cet acte poétique de mise en intrigue, ou plutôt dans ces actes, car il semble bien qu'il s'agisse en réalité d'une actio toujours recommencée, que se concrétise, pour saint Augustin le triple présent, sous la forme de la triple intentio. Mais si sur un plan purement spéculatif cette concordance des trois présents semble bien se réaliser, d'après Paul Ricouur (cf.op. cit, p. 49), justement à partir de leur discondance phénoménologique, sur le plan de la représentation mimétique elle semble non seulement improbable, mais aussi peu souhaitable, et elle engendre de toute façon une multitude de mises en intrigue qui intétessent le lecteur surtout pour leurs issues esthétiques.

${ }^{11}$ Mircea Eliade, Le mythe de l'étemel retour, Paris, Gallimard, 1969, p. 11.
} 


\section{Claudio GALDERISI}

mythe ${ }^{12}$ ", qui se prêtait aussi bien à des résurgences qu'à des réécritures, et en cela exemplaire de cette crise féconde de la mise en prose. On peut en effet reconnaître déjà dans son organisation diégétique tous les indices formels de ces nouvelles apories de la représentation romanesque. L'idée Mélusine, qui surgit des profondeurs du passé, se compose de mille fragments «emphatiquement signifiés ${ }^{13}$, plus ou moins perceptibles et reconnaissables sur le plan de l'enchaînement des signifiants, pour se transfigurer en personnage multiforme, qui pose au moins autant de problèmes sur un plan de l'histoire des idées que sur celui de sa genèse et de sa filiation narrative. Et d'ailleurs, la dualité semble bien être la nature profonde de ce mythe, comme peut-être de tout mythe, donc consubstantielle au personnage créé par Jean d'Arras en 1392.

Femme et serpente, bâtisseuse et destructrice, concrète parce que terrienne, mais aussi volatile comme le rêve dont elle est issue : diurne et nocturne, à la fois protectrice et mystérieuse, réelle et irréelle. Tout dans cette figure relève de la duplicité : double nature, double vie, double généalogie, symbole même de l'écriture qui se fait et se défait, d'un Moyen Age qui bâtit son image alors que celle-ci est en train de se dissoudre à tout jamais, aussi par cette même représentation. Rien de surprenant, alors, que seulement un siècle après que Jean d'Arras a " mis diligence de ceste histoire mettre en prose ${ }^{14}$ ", finisse par en naître deux récits distincts et pourtant spéculaires. Ce destin était déjà inscrit aussi bien dans ce que JeanJacques Vincensini définit avec une formule heureuse la translatio mythique ${ }^{15}$, ou la tentative de mettre en communication les dieux et les hommes, c'est-à-dire les hommes avec leur passé, que dans sa mise en intrigue ${ }^{16}$. Ainsi, même une simple mise en prose de "vrayes croniques ${ }^{17}$ " peut cacher et révéler à la fois comme dans un énorme catalyseur formel le choc de deux cultures et deux sociétés, que sous-tend déjà en partie le pluriel de «croniques».

On peut déjà remarquer, dans un premier temps, qu'il y a plusieurs différences structurelles entre le roman de Jean d'Arras et celui en vers de Coudrette, qui le suit de quelques années, aussi bien sur le plan de l'agencement des épisodes, que sur celui de leur statut diégétique. Coudrette a ainsi éliminé plusieurs récits des exploits de Geoffroy ${ }^{18}$, et cela malgré le fait qu'il ait écrit son roman en connaissant probablement le texte de Jean d'Arras ou

\footnotetext{
${ }^{12}$ Abbé Bullet, Dissertation sur Mélusine, Dissertations sur la mythologie française publiée en 1771, et reproduites dans Collection des meilleures dissertations relatives à l'Histoire de France, Paris, t. II, 1838 , p. 15 sq.

${ }^{13}$ Jean-Jacques Vincensini, Pensée mythique et narrations médiévales, Paris, Champion, 1996, p. 403.

${ }^{14}$ Jean d'Arras, Mélusine, éd. L. Stouff, Dijon, Publications de l'Université de Dijon (fasc. V), 1932, p. 308.

${ }^{15}$ Jean-Jacques Vincensini, op. cit., p. 239

${ }^{16}$ Peut-être conviendrait-il de parler de " mise en intrigue en prose ", puisque la version en vers de Coudrette, qui connaitra aussi un grand sucoès tout le long du XV siècle avec 20 manuscrits (cf. E. Roach, "La tradition manuscrite du roman de Mélusine par Coudrette ", Revue d'Histoire des textes, 7, 1977, pp. 185-233), ne sera pas en revanche à l'origine de deux romans autonomes.

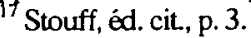

${ }^{18}$ Il supprime l'épisode breton, ainsi que les exploits en Bretagne et en Terre Sainte, ceux qui ont lieu en Autriche, le pèlerinage, et surtout ne dit pas mot du demier combat de Geoffroy avec le «chevalier fae».
} 
une autre version du récit" ${ }^{19}$. L'épisode de la découverte par Geoffroy à la grand dent des origines de sa lignée dans la montagne de Brumblerio en Northumberland, plaque tournante du système d'imbrication-dysjonction diégétique dans le roman de Jean d'Arras, Coudrette ne le place qu'à la fin de son récit, parce que le vers supporterait mal une tmèse narrative affaiblie par des révélations, alors que son objectif immanent, par delà le but politique, reste celui de créer un ailleurs cohérent et alternatif, une autre vie, où la redondance, l'aparté, n'entrent pas, faute de quoi toute la tension poétique s'essoufflerait. Mais ainsi faisant, il prolonge la symétrie romanesque des gestes de Mélusine-Raymondin et de Geoffroy jusqu'à l'explicit, niant de cette manière toute autonomie romanesque à l'un et à l'autre, et bouclant le projet narratif.

Ailleurs, dans le récit, nous avons un autre signe de cette divergence de stratégie et d'intérêts entre les deux récits. Chez Jean d'Arras, le duc de Poitiers, lors de la chasse au sanglier en compagnie de son neveu Raymondin, après une poursuite interminable, s'arrête le soir pour se reposer, et commence alors à regarder intensément le ciel étoilé, découvrant le secret de sa mort et de la fortune de celui qui le tuera dans sa famille. Exemple de disjonction rhématique, comme on peut en rencontrer dans tous les romans en prose du XIII siècle. Or, dans ce cas l'auteur de la mise en vers ne pouvait faire l'économie du passage, il a choisi alors le chemin du raccourci et de l'opacité : il dit sans tout dire, et surtout il annonce un événement qu'il ne met pas en relation avec les deux protagonistes, obligeant du coup Raymondin à un silence immotivé, qui jette une ombre inquiétante sur ses arrière-pensées. Jean d'Arras, lui, non seulement décrit avec plus de précision et de détails la vision du Duc, mais surtout selon le procédé bien connu de la disjonction, la met en relation avec les deux protagonistes, révélant de ce fait ce qui va se passer juste après cette vision.

Plus, Raymondin, à deux reprises, cherche à lutter contre son destin: une première fois quand il prie son oncle de ne pas se mêler des choses de l'astronomie : « Haa, monseigneur, par Dieu, laissiez telz choses ester. Il n'appertient point a si hault prince comme vous estes, mettre cure d'enquerre de telz ars, ne de telz choses ${ }^{20}$.» Puis, une fois échouée sa tentative de faire taire les astres, se refuse alors de croire à la vision qu'ils ont offerte au Comte de Poitiers: «Par foy, dist ly enfes, ce ne croiray je ja, car ce n'est mie chose qui face a croire $^{21} »$. Le personnage peut ainsi garder sa nature ambiguë de prédestiné et d'opportuniste.

Nous avons, donc, ce premier récit fondateur chez Arras, qui sert à établir une vérité qui précède le récit même, et dont l'authenticité ne découle pas de l'enchaînement narratif opéré par le narrateur, puisqu'elle est à la fois dans les "vrayes croniques » et le fait d'une volonté supérieure qui se manifeste au comte et à Raymondin, élément que renforce sa position à

\footnotetext{
${ }^{19}$ Roach pense, par exemple, que l'existence d'un modèle en vers français pour Coudrette " se déduit du fait, que, dans le texte de Coudrette, les fautes qui rendent faux certains vers ne peuvent s'expliquer que si l'on remonte à des formes soit verbales soit paléographiques d'une époque antérieure aux plus anciens manuscrits du texte " (E. Roach, art cit., p. 15).

${ }^{20}$ Stouff, éd. cit., p 20. Raymondin ne semble pas, lui, posséder cette science, privilège des nobles, et topos littéraire médiéval : “ Car, si comme dit l'ystoire, par ce temps nulz n'osoit faire apprendre ses enfans nul des VII. ars [...] s'ilz n'estoient nobles» (Stouff, éd. cit, p. 16). Et Jean de Salisbury de rappeler que "rex illiteratus asinus coronatus" (cf. Jean de Salisbury, Policraticus, éd. C. Webb, Oxford, $1909, \mathrm{r}, 6,524 \mathrm{~d})$.

${ }^{21}$ Ibid, p 21.
} 


\section{Claudio GALDERISI}

l'incipit. La story s'habille en history pour mieux faire passer sa verité. Certes, l'history concède très peu, surtout au XII et XII' siècles, aux effets et aux modes de la story, mais c'est le fait d'être écrite en langue romane et en prose qui en fait un événement littéraire en soi, et qui crée les conditions de l'union ${ }^{22}$, depuis indissoluble, entre récit et prose d'une part, narration et réalité d'autre part ${ }^{23}$. Une fois ce circuit établi, la correspondance entre réalité et vérité, entre réalité vécue et réalité subjective est pernicieusement, donc artistiquement, garantie par la similarité des structures qui les expriment et dont elles sont l'expression, et que seule la plus-value littéraire permet de distinguer ${ }^{24}$.

Mais c'est justement cette littérarité du texte qui pose problème aussi bien, on dirait, pour l'auteur in progress que pour ses lecteurs.

En effet, plusieurs critiques ont relevé une certaine négligence stylistique, et remarqué des incongruités temporelles, par delà les récurrences, tout à fait dans la norme, d'anachronies narratives ${ }^{25}$, dans le récit de Jean d'Arras. Ainsi, Geoffroy à la grand dent revenant de sa " mission » en Northumberland trouve ses deux frères Thierry et Raymonnet déjà adultes; toujours lors de sa rencontre avec le géant Grimaud, celui-ci faisant référence à l'affrontement avec l'autre géant, son cousin Gardon de Guérande, en parle comme s'il avait eu lieu depuis «quelques jours », alors qu'entre temps Geoffroy a pu aller aider ses frères en Palestine, visiter le saint sépulcre, mettre le feu à l'abbaye de Maillezais en tuant son frère Fromont, etc. Mais ces discordances temporelles, et l'absence, à nos yeux, de tout raffinement linguistique, sans parler des parataxes et des répétitions qui scandent comme une litanie métanarrative tout le récit, sont tout à fait courantes dans les récits en prose, et cela au moins jusqu'à la moitié du XV $v^{\ell}$ siècle. Ce qui est en revanche significatif est, d'une part, la maîtrise quasi parfaite de l'enchâssement narratif - dans certains agencements on serait même tenté de parler de fondu narratifif ${ }^{26}$, lorsque Jean d'Arras quitte le récit des aventures de Raymondin, pour revenir à Mélusine et inversement, et, d'autre part, les ruptures ${ }^{27}$ isotopiques

\footnotetext{
${ }^{22}$ Peut-être faudrait-il plutôt parler d'osmose structurale, puisque les structures du langage parlẻ nous renvoient l'image de la réalité vécue dont elles sont en partie le reflet.

${ }^{23}$ Madame Harf-Lancner rappelle, à ce propos, combien « culture savante et culture populaire semblent donc inextricablement liées dans la genèse du roman en prose " (Coudrette, Le Roman de Mélusine, éd. Laurence Harf-Lancner, Paris, GF-Flammarion, 1993, p. 24).

${ }^{24}$ Bernard Cerquiglini a subtilement analysé les modes de cette littérarité de la prose, rappelant que la * prose n'est pas un recours, le moyen d'échapper aux codes littéraires, elle ramène au contraire le chercheur au centre d'un jeu réglé de formes d'écritures, lui laissant entendre qu'il n'a pas d'autre objet." (B. Cerquiglini, La parole médiévale, Paris, Les Editions de Minuit, 1981, p. 18).

${ }^{25}$ Cf. Gérand Genette, Figures III, Paris, Seuil, 1972, p. 79sq.

${ }^{26}$ Dans cette partie du roman, en effet, la plupart des enchâssements semblent parfaitement validés, aussi bien sur le plan narratif que diégétique : « L'ystoire dit que entretant que Raymondin fut en Bretagne, Mellusine fist batir la ville de Lusignen et fonder les murs sur la vive roche, et la fit estoffer de fortes tours" (Stouff, éd. cit., p. 66). Suit, alors, une longue description de la citadelle faite bâtir par la fée, qui peut sembler gratuite, mais qui sert en réalité à préparer le retour du héros de sa valeureuse expédition en Bretagne, où il a fait preuve à la fois de courage et de largesse, distribuant à ses parents et amis toutes les terres et les biens de son héritage, enfin récupéré, parce que justement il recevra davantage de sa protectrice.

${ }^{27}$ Ou plutôt la rupture, parce que, une fois amivé sur le devant de la scène, le personnage de Geoffroy ne le quitte pratiquement plus jusqu'à la fin du roman, si l'on excepte l'épisode-clé, du moins pour le récit de Mélusine et de Raymondin, de la découverte de la vraie nature de la femme-fée, de la transgression
} 
qui marquent une vraie fracture diégétique entre le récit de Mélusine-Raymondin et celui des gestes de son fils Geoffroy ${ }^{28}$. Nous avons alors une structure diégétique du récit comparable, davantage, à celle ouverte, mais fondamentalement circulaire, des soap opéra, avec un collage réalisé par des opérations de couper-coller d'intertextes virtuels.

Certes, ailleurs, l'auteur fait aussi large preuve d'une remarquable capacité descriptive, avec une psychologie du détail qui s'exprime parfaitement dans les prises de vue des différentes batailles avec tout le bagage et le style de l'épopée, et avec un art des clairs-obscurs qui semble convenir parfaitement à la figure de Mélusine et à la ligne de fracture mythehistoire qui traverse le récit entier. L'auteur ne peut et ne veut pas, en revanche, éliminer les marques du métalangage, et continue d'agencer et de disloquer le récit de l'extérieur. C'est que d'une part son rôle de chroniqueur humble et fidèle lui semble mieux défendu par cette rupture de la fiction narrative, et que d'autre part il ne maitrise que rarement le fondu enchaîné, infradiégétique, qui représente un pas ultérieur vers la littéralisation de la mise en prose. Mais c'est aussi que le récit en prose privilégie cette structure fragmentée, qui correspond mieux au mode de pensée et de vie d'une société, pour laquelle le discontinu représente non seulement une esthétique, mais le mode de la vie et de sa pensée.

En effet, le caractère composite, la progression chaloupée semblent être constitutifs de toute écriture en prose, et l'intermittence de l'écriture ne peut pas être utilisée seule pour méconnaître l'unité romanesque du récit. S'il y a par moments glissement du récit vers le conte ou la nouvelle ${ }^{29}$ - l'épisode du château de l'Epervier, que les colporteurs ont cru nécessaire

des interdits de la part de Raymondin, et du conséquent envol de la serpente. (Stouff, éd. cit, pp. 25362). Mais ces pages vers lesquelles tend le roman de Mélusine dès son début, se révèlent comme marginales sur le plan narratif dans le récit des gestes de Geoffroy. C'est d'ailleurs le seul épisode signifiant, qui disparaitra dans les différentes éditions du Xví siècle du roman de Geoffroy à la grand dent, remplacé sur le plan diégétique, par la rencontre à Montjouet en Guérande, entre le héros de retour de son expédition en Northumberland et son frère Raymonnet, et qui servira à mettre au courant Geoffroy de la trahison de son oncle, le comte de Forez, de la disparition de sa mère, du deuil de son père. Un résumé métadiégétique, déjà présent dans le roman de Jean d'Arras, qui va permettre à Geoffroy de s'illustrer une fois de plus contre sa famille, par l'assasinat de son oncle.

${ }^{28}$ Nous avons, par exemple, à peine le temps, après la longue parenthèse narrative consacrée aux conquêtes des quatre premiers frères, de faire la connaissance de deux autres frères, Fromont et Thiérry, que l'auteur décide de les quitter pour l'aventure bien plus merveilleuse de Geoffroy : "Icy vous lerray a parler des deux enfants et vous parleray Gieffroy au grant dent [...] Car sachiez que cellui Gieffroy ne resoingna oncques hommes, et maintient l'ystoire et la vraye cronique que cellui Gieffroy se combati a ung chevalier fae ou au mauvais esperit es prez dessoubz Luseignen, et si comme vous orrez sy après en la vraye hystoire." (Stouff, éd. cit., pp. 196-97). En moins de dix lignes, à deux reprises, e par trois occurrences, Jean d'Arras met l'histoire de Geoffroy à la grand dent sous le double sceau de la vérité et de l'historicité, qui justifie toute rupture de l'artificielle linéarité narrative.

${ }^{29}$ On a rarement mis l'accent sur la place disproportionnée qu'occupent les récits des exploits des quatre fils-rois de Mélusine (Urien, Guion, Antoine et Renaud), reproduisant un schéma narratif et initiatique identique, parfaitement décrit par Sylvie Roblin (cf. «Le sanglier et la serpente, Geoffroy à la grand dent dans l'histoire des Lusignan ", Métamorphose et bestiaire fantastique au Moyen Age, Paris, ENSJF, 1985, pp. 247-85). Ces mini-séquences composent plus d'un tiers du roman (Stouff, ed. cit., pp. 67-196), sans que la littérature de colportage ait jamais pensé, toutefois, à en faire des romans, ou des contes à part entière. Mais ces exploits chevaleresques à la gloire des Lusignan, que justifiait aussi l'arrière-plan historique (mythe de la croisade à la fin du XIV siècle, etc.), par ailleurs devenu caduc et incompréhensible au XV siècle, se fondent sur la répétition du schéma qui « souligne son caractère topi- 
d'intégrer au récit des gestes de Geoffroy, en est peut-être l'exemple le plus évident-, ce n'est apparemment que du fait d'un rythme plus soutenu dans la présentation des personnages, ou de la mobilité et de la sveltesse des gestes où se résume le temps éternel de la préparation $^{30}$, lesquels ne sont que les symptômes narratifs d'une impossible weltanschauung romanesque. Sylvie Roblin a sans doute raison d'affirmer que «les aventures épiques formaient des excroissances sans lien organique avec le conte mélusinien proprement dit », et qu' " au lieu d'un récit continu et autonome, on a désormais avec Geoffroy une narration éclatée, croisée avec le conte : [...] indice structural que Geoffroy est bien celui qui provoque l'ébranlement dans l'histoire ${ }^{31}$. " Mais plus encore que de «narration éclatée», il conviendrait, peut-être, de parler de représentation éclatée ${ }^{32}$, entre, d'une part, l'histoire de Mélusine, conte type sur le mythe de l'éternel retour ${ }^{33}$, et d'autre part le récit des aventures de Geoffroy à la grand dent ${ }^{34}$, roman bref ou nouvelle longue, qui ne participe plus que par opposition, on dirait, de l'idéologie courtoise et chevaleresque, et qui appartient désormais tout entier à une esthétique du romanesque prémodeme. Michel Zink rappelait récemment, en parlant de la structure du récit et du rôle de l'entracte dans Méliador, que l'agglutination est une des

que et accentue, par contraste, l'individualisme de Geoffroy à la dent de sanglier, le plus «faé » des fils de Mélusine, le plus redoutable, le double masculin de la fée ", le plus romanesque " (L. Harf-Lancner, «Le Roman de Mélusine et le Roman de Geoffroy à la grand dent : les éditions imprimées de l'œuvre de Jean d'Arras », Bibliothèque d'Humanisme et Renaissance, L, 1988, p. 352).

${ }^{30}$ Dans son éclairant essai sur la « rapidité », tiré de ses « Norton Lectures », Italo Calvino cite l'histoire chinoise du sage Chouang-Tzu comme exemple de sédimentation et de maturation des sentiments et des pensées pour parvenir à une intuition instantanée épurée de toute impatience ou circonstance éphémère : «Entre autres nombreuses qualités, Chouang-Tzu avait une grande sûreté de main. Le roi lui demanda de dessiner un crabe. Chouang-Tzu dit qu'il lui fallait un délai de cinq ans, ainsi qu'une villa avec douze serviteurs. Au bout de cinq ans le dessin n'était pas commencé. «Il me faut cinq autres années ", dit Chouang-Tzu. Le roi les lui accorda. Quand s’acheva la dixième année, Chouang-Tzu prit le pinceau et en un instant, d'un seul trait, il dessina un crabe, le crabe le plus parfait qu'on eût jamais vu. ” (Italo Calvino, Leçons Américaines. Aide-mémoire pour le prochain millénaire, trad. de Yves Hersant, Paris, Gallimard, 1992, p. 93). Un motus in fine velocior, une accélération subite, constitutifs de la forme brève nouvelle, que l'on ne rencontre, en revanche, que très rarement dans ces récits discontinus et hoquetés.

${ }^{31}$ S. Roblin, op. cit, p. 258.

${ }^{32}$ Ou d'état de non-équilibre, qui en tant que résultant d'une contrainte macroscopique, la confection d'un objet littéraire, finit par être source d'ordre. (Voir, à ce propos, la passionnante analyse que font de l'état de non-équilibre d'une structure, et de la fonction de « créateur de compontements collectifs cohérents » de cette condition, Dya Prigogine et Isabelle Stengers dans Entre le temps et l'éternité, Paris, Flammarion, 1992, pp. 118-121.)

${ }^{33}$ Voir, à ce propos, le livre toujours actuel de Mircea Eliade, Le mythe de l'éternel retour, op. cit.

${ }^{34}$ Laurence Harf-Lancner observe une opposition entre "I'histoire de Mélusine... conte-type universel " et le « roman de chevalerie que constituent les aventures des dix fils de la fée... » (L. Harf-Lancner, art. cit. pp. 351-52). Mais le couple conceptuel conte-type universel - roman de chevalerie ne semble pas vraiment en antithèse. Les deux modèles, représentés ici par l'histoire de Mélusine et par celles de ses quatre fils-rois, participent des mêmes valeurs, ou pensées mythiques. Sur un plan idéologique, il n'y a aucune antinomie réelle entre les deux. Et si l'agencement narratif n'est pas toujours parfaitement maitrisé, leur arrière-plan diégétique est le même : celui du mythe en représentation historique. D'ailleurs, la littérature de colportage, comme nous le rappelions, ne s'y est pas trompée, qui n'a jamais séparé le mythe de la chevalerie, l'histoire de Mélusine de celle de ses quatre fils-rois, malgré la déliason structurale aussi opérante dans le texte de Jean d'Arras. 
conditions essentielles de la fiction romanesque ${ }^{35}$, et Stouff avait déjà signalé que Jean d'Arras avait pu se servir des deux rédactions de Méliador, parues vers 1365 et $1380^{36}$. Or, c'est justement ce remodelage idéologique et donc esthétique du mythe, aussi en action, par exemple, dans Méliador ${ }^{37}$, ou dans le Jehan de Saintré d'Antoine de la Salle, qui est à l'origine de cette mise en intrigue binaire du roman de Jean d'Arras.

Nous sommes peut-être là au cœur du lien complexe entre écriture, mise en prose, littéralisation et mythe. Si au début en effet l'écriture est au service du mythe pour construire son exemplarité, son éternité de mystère révélé, elle ne peut le faire que si, comme dans les textes sacrés, elle suit le schéma du récit sans se soumettre à aucune autre règle et contrainte que celle de dire la vérité selon les conditions imposées par le sacré. Selon la formule de Mircea Eliade: «le mythe est assumé par l'homme en tant qu'être total, il ne s'adresse pas seulement à son intelligence ou à son imagination ${ }^{38}$. " Autrement dit, il demande à être cru, acte de foi qui ne peut supporter au début aucun artifice externe. Ce n'est qu'ensuite, quand pour des raisons politiques, géographiques, dynastiques ou de simple désacralisation du modèle il n'est plus reçu comme révélation d'un mystère, épiphanie du sacré, se dégradant et se transformant en légende populaire, que les rôles se renversent et qu'alors, au service de l'écriture, il devient donc matière à fiction. C'est ici que la transgression de l'interdit inhérent à tout mythe assume une fonction métanarrative, sans laquelle il n'y aurait pas d'histoire, pas de mythe, pas d'auteur, pas de lecteurs. Qu'elle soit le fait de l'humain qui cède, ici Raymondin mu par sa jalousie ou par une curiosité originelle, donc interne au récit, ou d'un observateur exteme omniscient, la transgression est une fonction du récit, plus encore qu'un événement.

Mais puisque la story se fonde sur le mythe, cette langue ne pouvait pas être simplement celle de la communication, elle devait subir un procédé de raffinement, qui rendait sinon crédibles du moins interprétables les impossibilia, plus humaines, moins ésotériques, à travers enchaînements elliptiques et omissions, silences, dans un exercice de parfaite subjectivité littéraire.

Premier élément de ce travail de chroniqueur à l'inverse, donner au mythe, ici à celui de Mélusine, une dimension humaine, actualisant son histoire, par le baptême symbolique que constitue l'attribution d'un nom. A partir de ce moment-là, la fée a enfin un statut civil, elle est dans l'onymat, et peut faire partie de la société des humains. Ainsi, « le merveilleux meurt pour que l'on puisse enfin le reconnaitre ${ }^{39}$ \% mais il reste, toutefois, comme pôle culturel et idéologique du récit, son moteur et sa fin. Or, quand le contexte culturel et idéologique est

\footnotetext{
${ }^{35}$ Michel Zink, «Leçon au Collège de France », 19 mars 1995. A paraître prochainement dans les Actes du colloque Froissart, tenu à l'University of Massachusetts, novembre 1995.

${ }^{36}$ L. Stouff, Essai sur Mélusine, Dijon-Paris, Bellais-Picard, 1930, pp. 63-71.

${ }^{37} \mathrm{Il}$ est intéressant de signaler que dans certaines éditions du XVI ${ }^{\mathrm{e}}$ siècle de l'Histoire de Geoffroy à la grand dent, celle-ci est précédée justement de Méliador (cf. Les conquestes du tresnoble et raillant Geoffroy à la grand dent, seigneur de Lusignen et sisiesme filz de Melusine et de Raymondin, Conte dudict lieu, Paris, Jean Bonfons, s.d. [1543-1566], (Paris, B.N. Rés. Y² 678). L'état déplorable dans lequel se trouvent la plupart de ces éditions du $X^{e}{ }^{e}$ siècle m'a contraint à une brève consultation, qui ne m'a pas permis de vérifier toutes les modifications structurales et l'agencement des différents épisodes, comme il aurait été souhaitable. Pour les éditions du Roman de Mélusine du Xvié siècle (22), j'ai surtout consulté celle publiée à Paris, par Jean II Trepperel, vers 1526. (Paris, B.N. Rés. p Y ${ }^{2} 2788$ ).

${ }^{38}$ Mircea Eliade, Mythes, nêves et mystères, Paris, Gallimard, 1957, p. 14.

${ }^{39}$ S. Roblin, op. cit, p. 273.
} 


\section{Claudio GALDERISI}

connu d'avance, il explose ou plutôt implose sous le poids des contradictions, nous avons alors une fragmentation de la dimension psychologique, qui paraît alors incongrue, schizophrénique. Entre descriptions et repliements, le temps du récit progresse au début avec une lenteur qui convient à la dimension du roman et du mythe (festina lente), mais d'un seul coup tout se met à bouger autour de l'auteur, et le récit qui cherche à suivre de près cette nouvelle réalité psychologique se retrouve piégé dans différents projets, ou ébauches de projets narratifs. Il n'y a plus une seule issue possible, mais deux, trois, ou plus. Une incongruité psychologique qui mine l'unité narrative, affaiblit le contexte, qui de ce fait se décompose, se délie. Or c'est bien cette impossibilité de synthèse, cette impasse narrative, cette incapacité ou volonté d'alimenter l'intérêt du lecteur et de le guider de tous les points de la trame vers un dénouement narratif unique et précis, qui est en cause ici, et plus en général, dans les romans en prose du XIV et $X V^{e}$ siècles ${ }^{40}$. Et il faut, alors, qu'à chaque cicatrice diégétique corresponde une intrusion du translateur: «l'ystoire dit », «l'ystoire nous certiffie», "cy après la vraye histoire», etc., comme signe à la fois d'échec et de l'émergence d'un nouveau projet littéraire. Ces intrusions deviennent d'ailleurs systématiques chez Jean d'Arras, chaque fois qu'il revient à parler de Geoffroy à la grand dent.

Plus l'absence de modèles mythologiques se fait manifeste, plus s'affirme la nécessité d'un ancrage historique, mimétique, dans le mode de la translatio studii. Derrière la fiction romanesque commence à émerger une conscience nouvelle de la littérarité, de plus en plus liée à la fonction écrivain-roman-lecteur. Seulement pour s'affranchir de «l'illusion référentielle $^{41}$ ", pour exister, le romanesque ne peut pas ne pas bouleverser d'une part les fondements du rapport history-story et d'autre part les valeurs courtoises sur lesquelles se fonde la représentation lyrique de la société, à commencer évidemment par celui de la femme-muse. L'apparition du personnage Geoffroy n'implique pas la fin du monde merveilleux et chevaleresque, mythique, de Mélusine et de Raymondin, mais l'inadéquation de ce modèle, le leurre de la référentialité. De la même manière, l'apparition de damp Abbés, dans le Jehan de Saintré, bouleverse les codes de l'univers chevaleresque, et en interrompt le récit. Les aventures de Geoffroy à la grand dent n'ont rien ni d'une quête, malgré l'épisode de la découverte des origines de sa famille, ni d'une initiation amoureuse. Ce n'est pas seulement sa violence, son énergie impétueuse, son amour pour le combat - sa parole que l'on pourrait définir comme performative, parce que nécessaire à vaincre la force centripète du récit mélusinien - en un mot son exagération, qui en font un chevalier d'un nouveau genre ${ }^{42}$, c'est

\footnotetext{
${ }^{40}$ Certains passages dans le texte semblent même suggérer, sinon une conscience pleine de cette crise, du moins une acceptation du rôle et des tâches qui incombent à l'auteur : «...et, beaulx seigneurs, sachiez que qui jamais rien n'encommenceroit, jamais ne seroit nulle chose achevee. Il faut avoir en la chose commencement et moyen ainsi que la fin." (Stouff, éd. cit., p. 247). Or, c'est justement ce « moyen " qui pose problème. Car, une fois minée l'unité culturelle, donc représentative, le récit semble gouverné plus par une sénendipité diégétique, qui serait, d'ailleurs, la condition génétique d'un certain romanesque.

${ }^{41}$ Cf. M Riffaterre, L'illusion réferentielle, Littéraure et réalité, Paris, Seuil, 1982, pp. 91-118.

${ }^{42}$ En cinglant avec sa petite galère au secours de ses frères, Geoffroy arrive en plein déroulement de la bataille navale, et son enthousiasme et son envie de se battre sont tels qu'il en est presque aveuglé, finissant tout naturellement par oublier les devoirs envers sa famille : "Or alez, dist Gieffroy, mais, qui qu'ilz soient, je aideray a la plus faible partie, voire se ce ne sont mes freres»; pour tout de suite changer d'avis : “Or tost, dist Geoffroy, a eulx tost et appertement. ” (Stouff, éd. cit., pp. 217-18).
} 
aussi l'absence affichée de toute femme à ses côtés, y compris de sa mère Mélusine, qui n'apparait jamais avec lui dans le roman.

C'est qu'ayant perdu à la fois son statut de muse et d'être mythique, et ayant entrainé dans la nasse narrative du mariage et de la procréation son chevalier, Raymondin, la matière courtoise et chevaleresque, s'est réduite, condensée. Il ne reste plus que le conflit latent, et tout psychologique, de Raymondin avec lui-même, et de Mélusine avec son passé. Matière à drame, et une fois refroidie, aussi à tragédie. Mais ce serait franchement trop demander au simple translateur Jean d'Arras. Il est naturel que le flambeau du récit soit repris par la descendance. Et qui mieux, alors, qu'un chevalier courageux, intrépide à la force exceptionnelle, et capable de pervertir les canons de la tradition courtoise et chevaleresque, peut garantir la continuation du récit. Ainsi, le sanglier a condamné la serpente au silence, parce que celle-ci, en partie démythisée, soumise elle aussi, à un interdit, celui de sa condition d'épouse et de mère, est frappée d'impuissance romanesque, ne peut plus produire ou faire produire par son Raymondin d'aventures dignes de narration et d'intérêt. Il ne lui reste plus qu'à mettre en scène sa disparition scénographique, et au coeur du récit des gestes de Geoffroy, comme demier défi porté par la mère au fils qui l'a remplacée. Ce ne sont pas les funérailles du mythe de Mélusine, mais celles toujours pathétiques, aussi bien pour l'écrivain que pour son public, d'un personnage tant aimé, et désormais stérile, comme on en rencontre tous les jours dans les récits référentiels modernes : les feuilletons télévisés.

Les aventures de Geoffroy représentent aussi in absentia, alors, cette nouvelle conception du rôle de la femme-aphrodite à laquelle on a peut-être trop demandé, et dont l'image ne peut plus satisfaire ni l'auteur ni le lecteur médiéval ${ }^{43}$. Certes, l'émergence de cette tension qui relie monde réel et monde littéraire n'est pas en soi un fait nouveau ${ }^{44}$, mais elle est de plus en plus doublée d'une attention au début quasi inconsciente vers les lecteurs. Ce sont aussi ces qualités du texte, l'aséité esthétique des deux branches, qui ont dû attirer l'attention des colporteurs, jusqu'à faire du Roman de Mélusine et du Roman de Geoffroy à la grand dent des grands classiques populaires. Geneviève Bollème a parlé à propos de cette littérature d'une «écriture qui s'adapte à, se déforme vers, pour », et d'un public «qui à la fois demande et produit, commande et obéit ${ }^{45}$."

A travers le roman de Jean d'Arras, où modèle idéologique courtois et schéma mythique d'une part, goût du romanesque et réalisme expressif, d'autre part, se figent dans l'aporie diégétique de la non-représentabilité, modulée sur une double polarité esthétique, il apparait confusément mais sûrement que désormais la prose se donne pour objet de «remodeler le monde médiéval en fonction de l'attitude des lecteurs ${ }^{46} »$, mais surtout de mimer « la con-

\footnotetext{
${ }^{13}$ Désabusé, et de plus antiféministe, le clerc du $X V^{*}$ siècle se voit désormais contraint de mettre en représentation ce que Jankélévitch définira comme le "grand malentendu des sexes " (Vladimir Jankélévitch, Le Je-ne-sais-quoi et le Presque-rien 2. La méconnaissance. Le malentendu, Paris, Seuil, 1980, p. 203), obéissant alors à un nouveau précepte esthétique : "Dieu qu'il la fait bon dénigrer ".

${ }^{44}$ C'est justement dans ces mêmes années (1401-1402), que Christine de Pizan relance la polémique contre le Roman de la Rase de Jean de Meun, et que fleurit toute une production antiféministe, avec des textes comme Le Miroir de mariage d'Eustache Deschamps ou Les Quinze joies de mariage, etc., reprenant thèmes et motifs déjà largement exploités durant tout le XIV ${ }^{\mathcal{E}}$ siècle.

${ }^{45}$ Geneviève Bollème, La Bibliothèque bleue. La littérature populaire en France du XVf au XXX' siècle, Paris, Julliard, 1971, pp. 22 et 23.

${ }^{46}$ Moyen Age et colpontage, Robert le diable, éd. Lise Andries. Paris, Stock, 1981, p. 18.
} 


\section{Claudio GALDERISI}

frontation des humains avec l'insignifiance qui les entoure ${ }^{47}$.» Et s'il est vrai qu'une fois le genre codifié, "pour une seule et même narration, tous les registres de lecture sont envisagés dans l'unique intention de plaire au lecteur ${ }^{48} \%$, il est aussi vrai que dans une période de crise des genres, cette même intentio auctoris peut, comme dans le cas de L'Histoire de Geoffroy à la grand dent et celle de Mélusine ${ }^{49}$, se fractionner en deux ou plusieurs intentiones operis, formes néoplasiques de mimèsis, où se superposent et s'enchevêtrent plusieurs réseaux de registres de lecture, tous opérant pour une «seule narration», qui n'est plus forcément la même.

\section{Claudio Galderisi (Université de Mulhouse)}

\footnotetext{
${ }^{47}$ Jean-Jacques Vincensini, op. cit, p. 403.

${ }^{48}$ Roger Dragonetti, Le mirage des sources, Paris, Seuil, 1987, p. 265.

${ }^{49}$ Mais Mélusine n'est pas le seul récit à avoir donné lieu à deux romans distincts. Sans parler de l'exploitation des structures séquentielles en récits romanesques autonomes des différents épisodes du cycle du Lancelot-Graal, on peut citer, toujours au Xvi siècle, l'exemple de l'auteur énigmatique des Comptes du monde adventureux, qui composera à partir du Jehan de Saintré, trois nouvelles indépendantes (Cf. A.D.S.D., Les Comptes du monde adventureux, Paris, Estienne Groulleau, 1555 ; réédité par Félix Franck, Paris, Lemerre, 1878). Le cas apparemment inverse offert par la fusion des deux romans en vers le Bel inconnu et Jaufré, l'un en langue d'oìl, l'autre en langue d'oc, n'est dû en réalité qu'à une mise en prose tardive faite au XVI siècle par le remanieur Claude Platin. L'histoire de Giglan (le Bel Inconnu=Guinglain) et de Geoffroi de Maience (le Jaufré occitan), au titre d'ailleurs toujours binaire, est le résultat d'un entrelacernent artificiel, du moins sur le plan de l'organisation diégétique. Ainsi, deux siècles et demi après la première édition lyonnaise de 1520, le comte de Tressan n'hésita pas à rendre aux deux récits en prose leur liberté et leur indépendance narrative, en les insérant séparément dans la Bibliothèque universelle des romans (tome I, oct. 1777). Voir, à ce propos, la trés documentée mise au point de L. Harf-Lancner, «Le Bel inconnu et sa mise en prose au XV' siècle. L'histoire de Giglan: d'une esthétique à l'autre », Le chevalier et la menveille dans le Bel inconnu ou le Beau Jeu de Renaut, éd. J. Dufournet, Paris, Champion, 1996, pp. 70-89.
} 